\title{
Isolated Metacarpal Bone Metastasis from Advanced Rectosigmoid Carcinoma
}

\author{
Fadli AR, ${ }^{\mathrm{a}}$ Azmi $M N,{ }^{\mathrm{b}}$ David O, ${ }^{\mathrm{a}}$ Zailani $M H^{\mathrm{b}}$ \\ aDepartment of General Surgery, Hospital Tengku Ampuan Afzan, Kuantan, Pahang, Malaysia \\ ${ }^{b}$ Department of Surgery, Faculty of Medicine, International Islamic University, Kuantan, Pahang, Malaysia
}

\section{ABSTRACT}

Distant metastasis is a common sequelae of stage III colorectal adenocarcinoma. Liver and lungs are the most common sites for distant metastases, but, metastases to extremities are very rare. We report a case of rectosigmoid adenocarcinoma, which metastasizes to left first metacarpal bone, which is the first occurrence in our experience. The investigations and management of the patient are discussed.

KEYWORDS: Bone metastasis, colorectal carcinoma, osteolytic lesion, bone tumor.

\section{CASE REPORT}

A 67-year-old lady was diagnosed with Stage III rectosigmoid adenocarcinoma in early 2008. She underwent anterior resection and completed 12 cycles of FOLFOX (5-fluorouracil, oxaliplatin and folinic acid) regime as palliative chemotherapy. Unfortunately, she developed local recurrence and metastasis to the lung and liver 12 months after completing the chemotherapy. Furthermore, the level of carcinoembryonic antigen (CEA) was persistently elevated following the treatment. Despite these multi-organs metastases, she was relatively well and able to continue her normal daily life with assistance.

Two years after the diagnosis, she presented again with progressive painful swelling over the left thenar for two months in duration. She denied any prior history of trauma or similar swelling at other parts of the body. Physical examination showed a tender $3 \times 2 \times 2 \mathrm{~cm}$ bony swelling over her left thenar prominence with poorly defined margin. The overlyingskinwasnormal. Therewasnolimitation of the thumb movement and no distal neurovascular deficit. The plain radiological $x$-ray of left hand revealed osteolytic destruction of the first left metacarpal bone with no evidence of fracture. Fine-needle aspiration of the bony swelling confirmed the diagnosis of metastatic adenocarcinoma consistent with primary tumor from the gastrointestinal tract. The patient received symptomatic treatment for the pain.

Corresponding author:

Associate Professor Dr Mohd Zailani Mat Hassan

Department of Surgery, Faculty of Medicine, International Islamic University Malaysia, Jalan Hospital Campus, 25150 Kuantan, Pahang, Malaysia

Tel: +6095146090

Fax: +6095146090

H/P: +60122228764

Email: zailanih@yahoo.com

\section{DISCUSSION}

Colorectal carcinoma is one of the most common solid organ malignancies in the world and majority of them are adenocarcinoma. Distant metastasis is a common sequalae of colorectal adenocarcinoma, in particular, in Stage III diseases. Approximately $20 \%$ of patient will present with or develop the metastasis during the course of the disease. ${ }^{1}$ It can metastasize either hematogenously or via lymphatic drainage, although the former is more common.

Colorectal carcinoma metastases develop through a sequence of interrelated steps. Spreads to distant sites requires penetration of the vascular channel, resulting in either hematogenous or lymphatic channel spread. An international review of 1541 cases of colorectal cancers at autopsy demonstrated that hematogenous mestastasis evolves as a cascade, appearing first in the liver, followed by the lungs, before involving the other organs. ${ }^{2}$ This cascade is well illustrated in this case, where, before developing the metastasis to the metacarpal bone, she already had liver and lung metastasis. It means that the tumor embolus has passed through the portal circulation and the systemic circulation before deposited at the metacarpal bone.

Skeletal metastases are relatively uncommon in metastatic colorectal cancer. The incidence ranges from 1 to $7 \%$ diagnosed clinically and can be up to $20 \%$ in post mortem studies. ${ }^{3}$ Other uncommon sites for rectal metastasis which were reported in the literature include the palatine tonsils, breasts, urethra, mandible, thyroid and pancreas. ${ }^{2}$ The first report of a skeletal metastasis from rectal carcinoma was published in 1870 by Curling who described a metastasis to the radius. ${ }^{4}$ Subsequently, several other authors reported the involvement of other bones, including the digits. ${ }^{5,6}$ From our knowledge, metacarpal bone metastasis has never been reported in any literature. 


\section{IMJM}

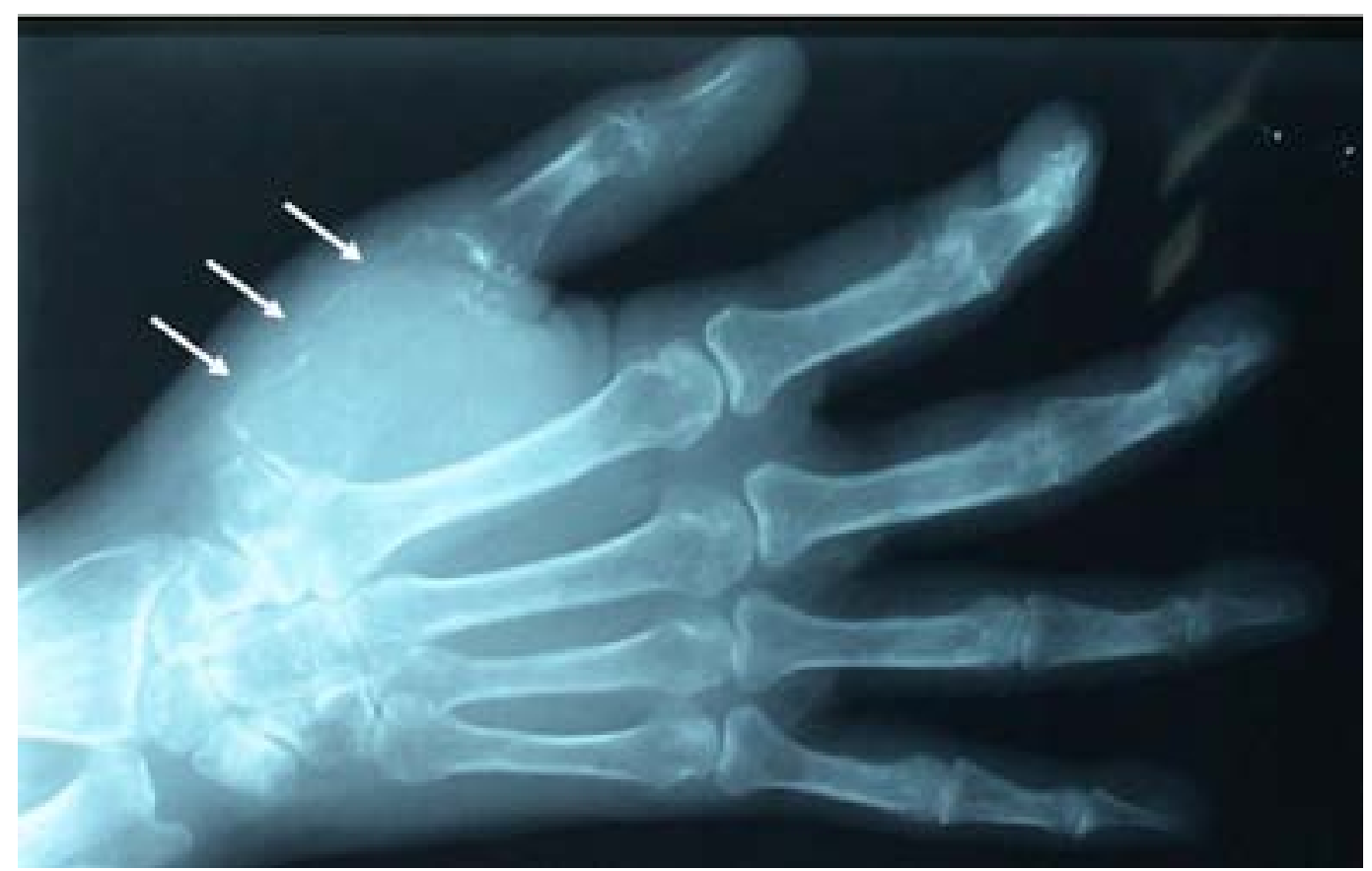

Figure I: Plain X ray of the left hand shows osteolytic destruction of the left first metacarpal bone

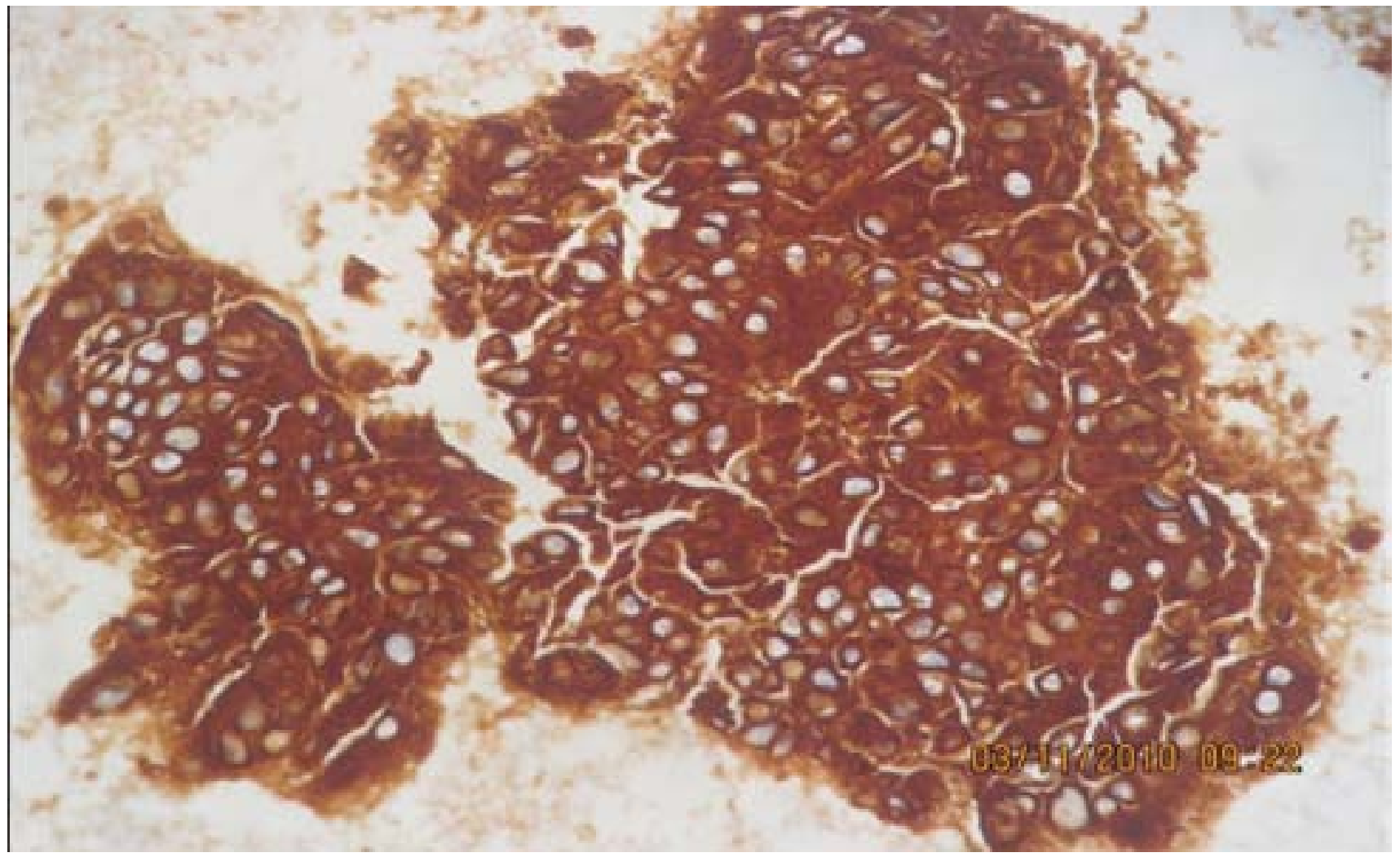

Figure 2: The cells showed high CEA positivity 
A history of previous malignancy is very important in making a diagnosis of metastasis to the extremities. Classically, there are five carcinoma sites with known tendencies to give rise to bone metastasis; breast, prostate, lungs, renal and thyroid but not from colon or rectum. ${ }^{7}$ Knowledge of this skeletal metastasis is very limited due to inadequate clinical data. Most patients presented with local bone pain, swelling or pathological fracture. Besbeas reported that bone metastases typically presented with multiple sites of bony skeletal involvement unlike in our patient. ${ }^{8}$ Routinely, imaging investigation of choice is a plain radiograph of the affected site. The appearance on the plain $x$-ray is usually osteolytic but mixed osteolytic-osteoblastic appearance is not rare which is associated with pseudo-sarcomatous appearance, with invasion of the soft tissue and a very marked periosteal reaction. ${ }^{9}$ Due to this osteolytic appearance, a diagnosis of a primary malignant lesion of the bone needs to be considered as well. Acomputerized tomography (CT) scan of the affected area may be helpful to demonstrate eccentric expansion of the bony cortex which is typically observed in metastasis. ${ }^{9}$ However, tissue biopsy is mandatory to confirm the diagnosis.

Skeletal metastasis leads to several complications such as severe pain, hypercalcaemia, pathological fracture and nerve root compression. Treatment options are only symptomatic relief, for instance, oral or intramuscular analgesia. If the pain is so severe, few fractions of external beam radiotherapy to the affected site is necessary. ${ }^{10}$

\section{CONCLUSION}

Isolated skeletal metastasis is uncommon in metastatic colorectal cancer and metacarpal metastasis has never been reported in the literature. Diagnosis requires a high index of suspicion as the incidence is extremely rare. It should be suspected in patients with advanced colorectal cancer presented with progressive bony swelling. Diagnosis is confirmed with radiological imaging and tissue biopsy. Palliative management is usually sufficient as the prognosis is relatively poor.

\section{REFERENCES}

1. Cook $A D$, Single R, McCahill LE. Surgical resection of primary tumors in patients who present with stage IV colorectal cancer: an analysis of surveillance, epidemiology and end results data, 1988 to 2000. Ann Surg Oncol 2005;12:637-45.

2. Weiss L, Grundman E, Torhorst J, et al. Hema togenous metastatic patterns in colonic carcinoma: an analysis of 1541 autopsies. J Pathol 1986;150:195-203.

3. Sebag-Montefiore DJ, Lam KS, Arnott SJ. Case report: Tarsal metastases in a patient with rectal cancer. Br J Radiol 1997;70: 862-4.

4. Curling TB. Case of cancerous stricture of the rectum producing obstruction, successfully relieved by colostomy. Lancet $1870 ; 1: 3$

5. Bacon HE, Jackson CC. Skeletal metastases from carcinoma of the rectum. Can Med Assoc J 1954;70:189-91.

6. Buckley N, Peebles Brown DA. Metastatic tumors in the hand from adenocarcinoma of the colon. Dis Colon Rectum 1987;30:141-3.

7. Ogilvie CM., Fox EJ, Lackman RD. Current surgical management of bone metastases in the extremities and pelvis. Semin Oncol 2008;35:118-28.

8. Besbeas S, Stearns MW. Osseous metastases from carcinoma of the colon and rectum. Dis Colon Rectum 1978;21:266-8.

9. Oh YK, Park HC, Kim YS. Atypical bone metastasis and radiation changes in a colon cancer: A case report and a review of the literature. Jpn J Clin Oncol 2001;31:168-71.

10. Coleman RE. Management of bone metastses. Oncologist 2000;5:463-70. 
THE INTERNATIONAL MEDICAL JOURNAL MALAYSIA
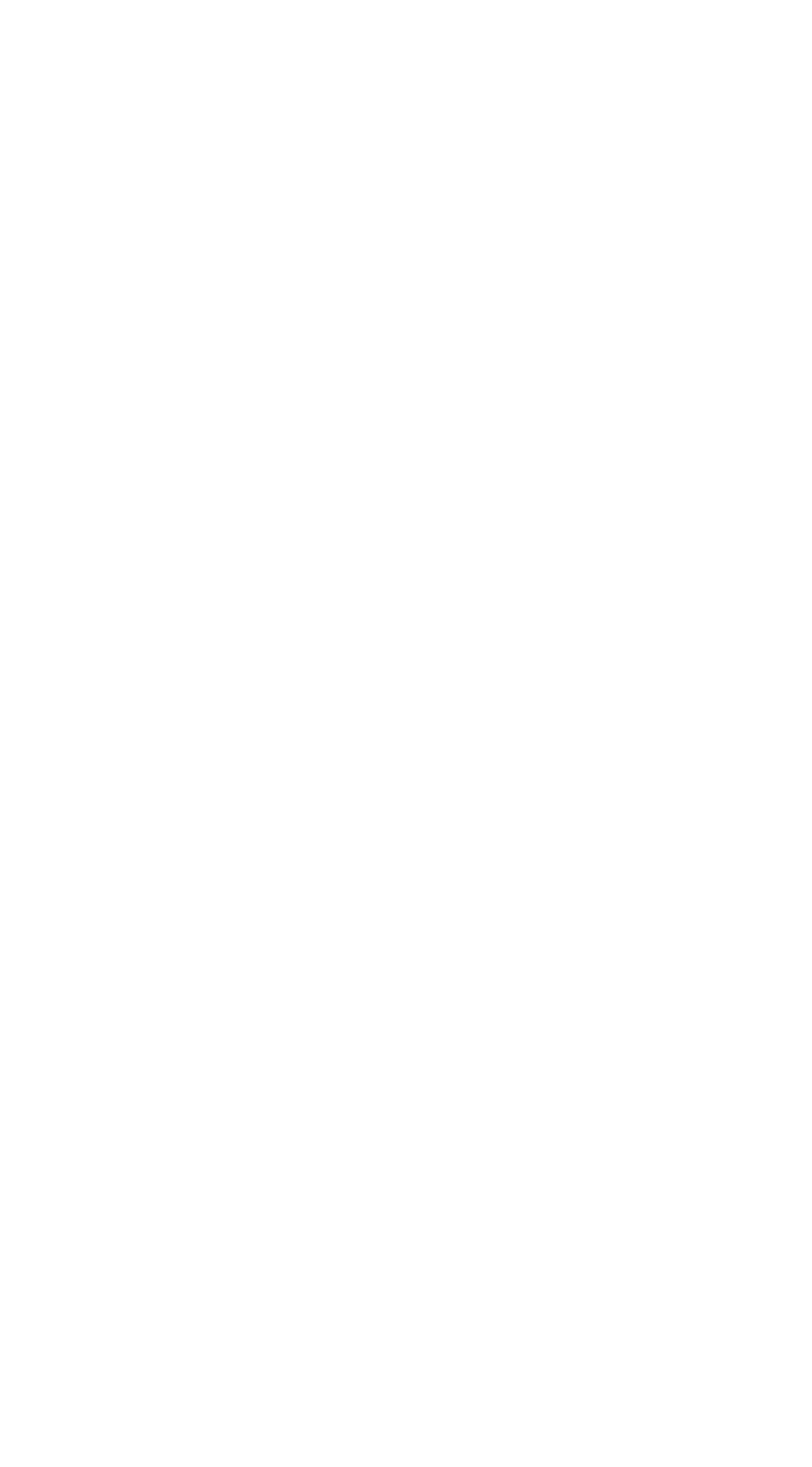